\title{
Beneficial effects of dietary restriction in type 2 diabetic rats: the role of adipokines on inflammation and insulin resistance
}

\author{
Joana Crisóstomo ${ }^{1,2} *$, Lisa Rodrigues ${ }^{1,2}$, Paulo Matafome ${ }^{1,2,3}$, Carmen Amaral ${ }^{1}$, Elsa Nunes ${ }^{1}$, \\ Teresa Louro ${ }^{1,2}$, Pedro Monteiro ${ }^{2,4}$ and Raquel Seiça ${ }^{1,2}$ \\ ${ }^{1}$ Faculty of Medicine, Institute of Physiology, University of Coimbra, Coimbra, Portugal \\ ${ }^{2}$ Faculty of Medicine, Institute of Biomedical Research in Light and Image, University of Coimbra, Coimbra, Portugal \\ ${ }^{3}$ Faculty of Medicine, Centre of Ophthalmology, IBILI, University of Coimbra, Coimbra, Portugal \\ ${ }^{4}$ Basic Research Unit in Cardiology, Cardiology Department, Coimbra University Hospital and Medical School, Coimbra, \\ Portugal
}

(Received 26 June 2009 - Revised 8 December 2009 - Accepted 11 January 2010 - First published online 24 February 2010)

\begin{abstract}
Inflammation plays an important role in diabetes mellitus and its complications. In this context, the negative cross-talk between adipose tissue and skeletal muscle leads to disturbances in muscle cell insulin signalling and induces insulin resistance. Because several studies have shown that energy restriction brings some benefits to diabetes, the aim of the present study was to evaluate the effects of dietary restriction on systemic and skeletal muscle inflammatory biomarkers, such C-reactive protein, adipokines and cytokines, and in insulin resistance in Goto-Kakizaki rats. This is an animal model of spontaneous non-obese type 2 diabetes with strongly insulin resistance and without dyslipidaemia. Animals were maintained during 2 months of dietary restriction $(50 \%)$ and were killed at 6 months of age. Some biochemical determinations were done using ELISA and Western blot. Data from the present study demonstrate that in Goto-Kakizaki rats the dietary restriction improved insulin resistance, NEFA levels and adipokine profile and ameliorated inflammatory cytokines in skeletal muscle. These results indicate that dietary restriction in type 2 diabetes enhances adipose tissue metabolism leading to an improved skeletal muscle insulin sensitivity.
\end{abstract}

Type 2 diabetes: Adipokines: Insulin resistance: Inflammation: Dietary restriction: Skeletal muscle

Genetic and environmental factors such as sedentary lifestyle and increased energy consumption are associated with overweight, which is a major risk factor for type 2 diabetes. According to the WHO, at least 171 million individuals worldwide are affected and the prevalence will be dramatically increased over the coming decades ${ }^{(1)}$.

Inflammation leads to diabetic complications, being intimately linked with insulin resistance and the atherogenic process which may result in cardiovascular and cerebrovascular diseases ${ }^{(2-8)}$. Understanding the mechanisms and regulation of inflammatory pathways can be an important key to reduce diabetes-associated problems.

Cytokines such as TNF- $\alpha$ and IL-6, lipids, intracellular stress and excessive reactive oxygen species production are extracellular mediators enabling the initiation of inflammatory pathways ${ }^{(3)}$. Signals from all of these mediators come together to cause the production of additional inflammatory mediators through transcriptional regulation. These inflammatory signalling pathways stimulate a number of different kinases, including the kinase Jun-N-terminal kinase (JNK) that induces serine phosphorylation of insulin receptor substrate- $\mathrm{I}^{(2,3,5,7,9)}$.
In fact, JNK plays a role in the negative regulation of insulin signalling, but the contribution of this pathway in adipose tissue, skeletal muscle or other tissue to systemic insulin resistance is currently unclear. Recent studies in mice reported that JNK inhibition in diabetes or atherosclerosis can be a viable therapy for these diseases in humans ${ }^{(3)}$.

Skeletal muscle is responsible for over $75 \%$ of all insulinmediated glucose disposal ${ }^{(8)}$. Therefore, mechanisms of glucose homeostasis are particularly important in this tissue. It is now believed that a negative cross-talk between adipose tissue and skeletal muscle, through adipokines, leads to disturbances in skeletal muscle insulin signalling and finally to insulin resistance, which seems to involve inflammatory components $^{(2,3,5,7,8,10-13)}$.

Studies in a variety of species have shown that energy restriction brings some benefits to health. Rhesus monkeys submitted to energy restriction are less susceptible to CVD and diabetes mellitus ${ }^{(14)}$. Energy restriction in adult men and women causes many of the same metabolic adaptations that occur in energy-restricted rodents and monkeys, such as decreased metabolic, hormonal and inflammatory risk factors

Abbreviations: GK, Goto-Kakizaki; GKC, Goto-Kakizaki control; GKDR, Goto-Kakizaki rats with dietary restriction; HbA1c, glycated Hb; HOMA, homeostasis model assessment; IRS, insulin receptor substrate; JNK, Jun-N-terminal kinase; QUICKI, quantitative insulin-sensitivity check index; SAPK, stress-activated protein kinase; WC, Wistar control; WDR, Wistar rats with dietary restriction.

* Corresponding author: Dr Joana Crisóstomo, fax +351 239 480034, email joanacrisostomo@hotmail.com 
for diabetes, CVD and possibly cancer ${ }^{(15)}$. Accordingly, the characteristic up-regulation of inflammatory pathways in diabetes has also been reported to decrease with diet intervention in streptozotocin-induced diabetic rats ${ }^{(6)}$. Furthermore, the guidelines of the American Diabetes Association highlight the importance of medical nutrition therapy, not only for prevention, but also for metabolic control and for delaying complications of this pathology ${ }^{(16)}$.

As the impairment in skeletal muscle actions may have serious effects on whole-body glucose homeostasis, the aims of the present study were to evaluate the effects of dietary restriction on systemic and skeletal muscle inflammatory signals in Goto-Kakizaki (GK) rats, and understand the role of adipose tissue in those pathways. Therefore, the last aim was to inquire about dietary restriction as a therapeutic complement to diabetes treatment.

In fact, several studies about nutritional intervention, namely energy restriction, have been done in type 2 diabetes. In the present study, rats were submitted to $50 \%$ of dietary restriction. This approach makes a nutritional intervention easier, because the restriction did not consider the nutritional nature of the food, but the amount of food ingested. The peculiarity of GK rats also shows a new contribution in this area, because this diabetic animal model is non-obese and, consequently, the benefits shown of dietary restriction occurred outside the loss of body weight.

We demonstrated that GK rats are strongly insulin resistant, which implies large alterations in glucose metabolism in this tissue. Our data also demonstrate that our animal model has impaired adipokine physiology, although it does not have obesity or dyslipidaemia. Thus, the present study primarily shows that alterations of adipokines and insulin action are strong allies in impairing glucose metabolism in the skeletal muscle, where inflammatory pathways seem to be involved.

\section{Materials and methods}

\section{Experimental animals}

Male normal Wistar and GK (an animal model of spontaneous non-obese type 2 diabetes) rats were obtained from our local breeding colonies (Faculty of Medicine, University of Coimbra, Coimbra, Portugal).

Animals were maintained under controlled light $(12 \mathrm{~h}$ light $-12 \mathrm{~h}$ dark cycle), temperature $\left(21-24^{\circ} \mathrm{C}\right)$ and humidity (50-60\%) conditions and with free access to water and a standard diet (PanLab S.I. Barcelona, Spain). Rats were separated into four experimental groups. Two groups - Wistar control (WC) and GK control (GKC) - were maintained on the standard diet for 6 months (chow intake of 26 and $24 \mathrm{~g} / \mathrm{rat}$ per $\mathrm{d}$, respectively). The other two groups were maintained on dietary restriction (Wistar dietrestricted (WDR) and GK diet-restricted (GKDR), at 50\% of control animals' daily chow intake) during the last 2 months of life (chow intake of 13 and $12 \mathrm{~g} / \mathrm{rat}$ per $\mathrm{d}$, respectively). When the study finished, all animals were aged 6 months.

All animal procedures and care were approved by the local Institutional Animal Care and Use Committee.

\section{Sample collection and in vivo determinations}

At the end of diet intervention (6 months of age), rats were fasted overnight in order to measure blood glucose levels. Fasting blood glucose and at $2 \mathrm{~h}$ after glucose load $(1.8 \mathrm{mg}$ glucose $/ \mathrm{kg}$ ) were measured on the tail vein through the glucose-oxidase method using a glucometer (Elite; Bayer, Carnaxide, Portugal) and compatible reactive test strips.

Glycated $\mathrm{Hb}(\mathrm{HbA} 1 \mathrm{c})$ was also measured in the tail vein blood using an automatic analyser (DCA 2000; Bayer).

For further determinations, fasting blood samples were collected by cardiac puncture from anaesthetised animals with ketamine chloride $(75 \mathrm{mg} / \mathrm{kg}$, intramuscular; ParkeDavis, Ann Arbor, MI, USA) and chlorpromazine chloride $(2.65 \mathrm{mg} / \mathrm{kg}$, intramuscular; Laboratórios Vitória, Amadora, Portugal). For the determination of plasma and serum parameters, blood was centrifuged $(2500 \mathrm{rpm})$ at $4^{\circ} \mathrm{C}$ and stored at $-20^{\circ} \mathrm{C}$.

\section{Muscle removal}

Rats were killed by cervical displacement and skeletal muscle from the posterior face of the femur was removed and immediately frozen in liquid $\mathrm{N}_{2}$ and stored at $-80^{\circ} \mathrm{C}$.

\section{Insulin resistance indexes and NEFA}

Plasma insulin was determined by an in-house competitive ELISA $^{(17)}$. Insulin resistance was evaluated through the log-homeostasis model assessment (HOMA) index and the quantitative insulin-sensitivity index (QUICKI). Log-HOMA was calculated as:

$$
\log -\text { HOMA }=\text { logarithm of }\left(\left(\mathrm{I}_{\mathrm{f}}\right) \times\left(\mathrm{G}_{\mathrm{f}}\right)\right) / 22 \cdot 5,
$$

where $\left(I_{f}\right)$ is the fasting insulin level $(\mu U / m l)$ and $\left(G_{f}\right)$ is the fasting glucose level $(\mathrm{mmol} / \mathrm{l})$. QUICKI was calculated as $1 /\left(\log \left(\mathrm{G}_{0}\right)+\log \left(\mathrm{I}_{0}\right)\right)$, where $\mathrm{G}_{0}$ is fasting glucose $(\mathrm{mg} / \mathrm{dl})$ and $I_{0}$ is fasting insulin $(\mu \mathrm{U} / \mathrm{ml})$.

Plasma NEFA levels were measured using a commercial kit (Half-micro test; Roche Diagnostic, Mannheim, Germany).

\section{Determination of systemic inflammatory biomarkers}

Serum C-reactive protein levels were measured using the C-reactive protein ELISA kit (BD Biosciences Pharmingen, San Diego, CA, USA).

Serum leptin and adiponectin levels were measured by a Leptin Immunoassay kit (Invitrogen, Carlsbad, CA, USA) and an ELISA Phoenix Adiponectin Kit Assay (Phoenix Pharmaceuticals Inc., Belmont, CA, USA), respectively.

\section{Muscle analysis}

Frozen skeletal muscle sections were homogenised in a lysis buffer containing $25 \mathrm{~mm}$-2-amino-2-(hydroxymethyl) propane-1,3-diol (Tris)- $\mathrm{HCl}, 100 \mathrm{~mm}-\mathrm{NaCl}, 1 \mathrm{~mm}$-EDTA, $1 \mathrm{~mm}$-ethylene glycol-bis(2-aminoethyl)-N,N,N $\mathrm{N}^{\prime}, \mathrm{N}^{\prime}$-tetraacetic acid, $20 \mathrm{~mm}-\mathrm{NaF}, \quad 10 \mathrm{~mm}-\beta$-glycerolphophate, $\quad 2.5 \mathrm{~mm}$ pyrophosphate, $2 \mathrm{~mm}_{-} \mathrm{Na}_{3} \mathrm{VO}_{4}, 1 \%$ Triton $\mathrm{X}-100,10 \mathrm{~mm}-$ phenylmethanesulfonyl fluoride and protease inhibitor cocktail (Sigma, St Louis, MO, USA) (pH 7.4). Homogenates were 
centrifuged at $14000 \mathrm{rpm}$ for $20 \mathrm{~min}$ at $4^{\circ} \mathrm{C}$. The supernatant fraction was collected and transferred to a clean tube and centrifuged at $14000 \mathrm{rpm}$ for $15 \mathrm{~min}$ at $4^{\circ} \mathrm{C}$ and the supernatant fraction was collected again. Protein concentration was determined by the Biureth method.

Samples were stored at $-80^{\circ} \mathrm{C}$ for subsequent analysis.

Muscle TNF- $\alpha$ and IL- 6 levels were determined using a Rat TNF ELISA Set and a Rat IL-6 ELISA Set (BD Biosciences Pharmingen), respectively.

The expression of stress-activated protein kinase (SAPK)/ JNK and phospho-SAPK/JNK was determined by the Western blot method. Samples containing $40 \mu \mathrm{g}$ protein were loaded into $10 \%$ acrylamide gels and transferred to Immun-Blot ${ }^{\circledR}$ polyvinylidine difluoride membranes. The membranes were blocked with $5 \%$ bovine serum albumin-TBST solution (25 mM-Tris- $\mathrm{HCl}, 150 \mathrm{mM}-\mathrm{NaCl}(\mathrm{pH} \mathrm{7.6)}$ and $0.1 \%$ Tween) at room temperature for $1 \mathrm{~h}$ and then washed with TBST solution. After that, membranes were incubated overnight at $4^{\circ} \mathrm{C}$ with a specific primary antibody of SAPK/JNK (SAPK/ JNK rabbit polyclonal antibody; Cell Signaling Technology, Danvers, MA, USA). The membranes were washed and incubated with a secondary antibody for $2 \mathrm{~h}$ at room temperature (anti-rabbit IgG alkaline phosphatase-linked goat antibody; GE Healthcare, Slough, Berks, UK). Membranes were washed and the immunoblots were developed with an ECF Western blotting detection system (STORM 860; GE Healthcare Life Sciences, Piscataway, NJ, USA). The same protocol was carried out for phospho-SAPK/JNK (phosphoSAPK/JNK (Thr183/Tyr185) mouse monoclonal antibody; Cell Signaling Technology).

\section{Reagents}

All the other compounds were of the purest quality available and were purchased from Sigma Chemical Co. (St Louis, MO, USA) or Merck (Darmstadt, Germany).

\section{Statistical analysis}

Data are presented as mean values with their standard errors. Statistical differences were determined by Student's $t$ test. Results with $P<0.05$ were considered statistically significant.

\section{Results}

Body weight, glycaemia and glycated $\mathrm{Hb}$

As previously reported, adult diabetic GK rats of our colony were smaller than the corresponding control Wistar rats ${ }^{(18)}$. Accordingly, the body weight of diabetic rats was significantly lower $(P<0.001)$ and was maintained after dietary restriction, although a great reduction was observed in visceral adipose tissue. A significant decrease in body weight $(P<0 \cdot 001)$ was observed in the WDR group (Table 1). Glycaemia, fasting and $2 \mathrm{~h}$ after glucose load, was significantly elevated $(P<0.001)$ in GKC rats when compared with normal rats (WC group). After dietary restriction, fasting glycaemia increased in both groups $(P<0.01)$ and glycaemia at $2 \mathrm{~h}$ decreased in the GKDR group $(P<0.05)$ (Table 1$)$. HbA1c levels were higher in GKC rats when compared with agematched Wistar rats (WC group) $(P<0.001)$ and decreased in both groups $(P<0.001)$ after dietary restriction (WDR and GKDR groups; Table 1).

\section{Dietary restriction improves insulin resistance indexes and NEFA levels}

The insulin resistance index log-HOMA (Fig. 1(a)) was higher and QUICKI, an insulin-sensitivity index (Fig. 1(b)), was lower in GKC rats, when compared with normal Wistar (WC) rats $(P<0 \cdot 001)$. Dietary restriction was able to improve insulin resistance $(P<0.01)$ and sensitivity $(P<0.001)$ in the diabetic rats (GKDR group).

Dietary restriction decreased plasma NEFA in both experimental groups $(P<0 \cdot 05)$. GKDR group levels were lower $(P<0.01)$ than those in the WC group (Table 1$)$.

\section{Systemic inflammatory biomarkers}

Levels of serum C-reactive protein, a systemic inflammatory marker, were significantly reduced in GKC rats $(P<0 \cdot 001)$, when compared with normal Wistar group (WC) values and were not changed with dietary restriction in both strains (Fig. 2).

Serum levels of leptin were significantly increased $(P<0.01)$ in GKC rats, when compared with normal Wistar (WC) rats. Dietary restriction decreased leptin levels in both strains $(P<0 \cdot 001)$ (Fig. 3(a)).

Table 1. Body weight, fasting glycaemia, glycaemia at $2 \mathrm{~h}$ after intraperitoneal glucose administration $(1.8 \mathrm{~g} / \mathrm{kg})$ and glycated $\mathrm{Hb}(\mathrm{HbA} 1 \mathrm{c})$ and NEFA levels in control and dietary restriction groups

(Mean values with their standard errors for nine to thirteen rats per group)

\begin{tabular}{|c|c|c|c|c|c|c|c|c|}
\hline \multirow[t]{2}{*}{ Group... } & \multicolumn{2}{|c|}{ wC } & \multicolumn{2}{|c|}{ GKC } & \multicolumn{2}{|c|}{ WDR } & \multicolumn{2}{|c|}{ GKDR } \\
\hline & Mean & SEM & Mean & SEM & Mean & SEM & Mean & SEM \\
\hline Body weight (g) & $403 \cdot 9$ & $6 \cdot 9$ & $312 \cdot 7^{\star \star \star}$ & $5 \cdot 8$ & $336 \cdot 6^{\star \star *}$ & 7.5 & $303 \cdot 9^{\star \star \star}$ & 3.7 \\
\hline Fasting glycaemia (mg/l) & 652 & 20 & $931^{\star \star *}$ & 25 & $742^{\star *}$ & 12 & $1088^{* * \star}+\dagger \dagger$ & 28 \\
\hline Glycaemia at $2 \mathrm{~h}(\mathrm{mg} / \mathrm{l})$ & 820 & 26 & $2994^{\star \star \star}$ & 72 & 775 & 26 & $2538^{\star \star \star} \dagger$ & 289 \\
\hline HbA1c (\%) & 3.5 & 0.0 & $6 \cdot 1^{\star \star \star}$ & 0.4 & $3 \cdot 1^{\star \star \star}$ & 0.0 & $4 \cdot 0^{\star \star *}+\dagger \dagger$ & 0.1 \\
\hline NEFA (mm) & 0.7 & 0.1 & 0.8 & 0.0 & $0.5^{\star}$ & 0.0 & $0.6^{\star \star} \dagger$ & 0.1 \\
\hline
\end{tabular}

WC, Wistar control; GKC, Goto-Kakizaki control; WDR, Wistar rats with dietary restriction; GKDR, Goto-Kakizaki rats with dietary restriction.

Mean value was significantly different from that of the WC group: * $P<0.05,{ }^{* *} P<0.01,{ }^{* * *} P<0.001$

Mean value was significantly different from that of the GKC group: $† P<0.05$, $\uparrow \dagger \uparrow P<0.001$. 

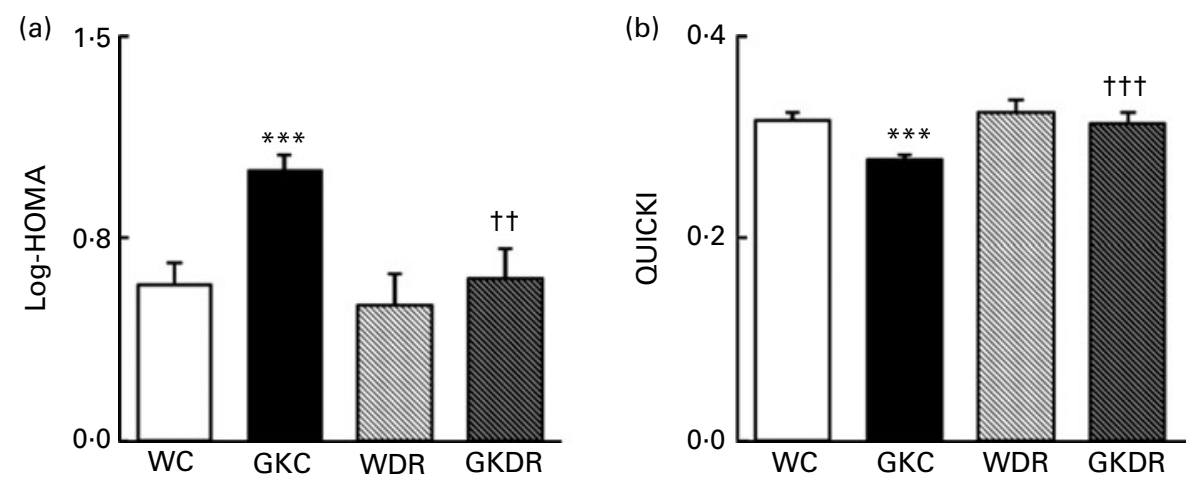

Fig. 1. Effects of dietary restriction on (a) the homeostasis model assessment index (HOMA; an index of insulin resistance) and (b) the quantitative insulinsensitivity check index (QUICKI) in Wistar and Goto-Kakizaki (GK) rats. WC, Wistar control; GKC, GK control; WDR, Wistar rats with dietary restriction; GKDR, GK rats with dietary restriction. Values are means for nine to thirteen rats per group, with standard errors represented by vertical bars. ${ }^{\star * \star}$ Mean value was significantly different from that of the WC group $(P<0.001)$. Mean value was significantly different from that of the GKC group: $\dagger \dagger P<0.01, \dagger \dagger \dagger P<0.001$.

Serum levels of adiponectin were lower in GKC rats when compared with age-matched normal WC rats $(P<0 \cdot 05)$. The dietary restriction improved these levels in WDR and GKDR groups $(P<0.05$ and $P<0.01$, respectively). Furthermore, GKDR adiponectin levels were similar to the values of normal (WC and WDR) rats (Fig. 3(b)).

\section{Muscular inflammatory biomarkers}

Muscular levels of TNF- $\alpha$ were significantly reduced $(P<0.001)$ in GKDR rats when compared with GKC and WC values (Fig. 4(a)).

The levels of IL-6 were higher in GKC rats $(P<0 \cdot 05)$ when compared with those of the WC group and decreased in the GKDR group $(P<0 \cdot 05)$. In contrast, dietary restriction induced an increase $(P<0.05)$ of IL-6 values in normal Wistar (WDR) rats (Fig. 4(b)).

Dietary restriction decreases phospho-stress-activated protein kinase/Jun-N-terminal kinase in diabetic rats

According to some studies which postulated that inflammation in diabetes mellitus can be related to the activity of the active

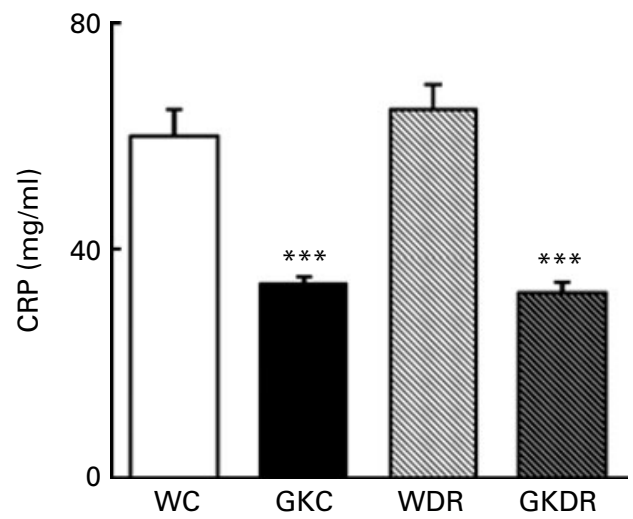

Fig. 2. Effects of dietary restriction on serum levels of C-reactive protein (CRP) in Wistar and Goto-Kakizaki (GK) rats. WC, Wistar control; GKC, GK control; WDR, Wistar rats with dietary restriction; GKDR, GK rats with dietary restriction. Values are means for nine to thirteen rats per group, with standard errors represented by vertical bars. ${ }^{\star \star \star}$ Mean value was significantly different from that of the WC group $(P<0.001)$. form of SAPK/JNK, the expression of normal and phosphorylated forms of this protein was measured. As illustrated by representative images and quantitative analysis (Fig. 5), the expression of SAPK/JNK was similar in the four groups studied (Fig. 5(a) and (b)). Nevertheless, the levels of the active phospho-SAPK/JNK form decreased in GKDR rats $(P<0.05)$, when compared with the GKC and WC groups (Fig. 5(c) and (d)).

\section{Discussion}

Data from the present study demonstrated that GK rats, a model of type 2 diabetes, compared with normal control rats, exhibited fasting hyperglycaemia and glucose intolerance, higher levels of $\mathrm{HbAlc}$ and insulin resistance. Serum adiponectin was lower and plasma leptin was higher, demonstrating adiponectin deficiency and the previously reported leptin resistance in this animal model ${ }^{(19)}$. These results are also indicative of adipocyte dysfunction already observed in diabetes mellitus and in insulin-resistant states ${ }^{(20)}$. Serum levels of C-reactive protein were lower in GK rats when compared with normal rats. These results show an intrinsic characteristic of this strain that was previously observed from our group (J Crisóstomo, L Rodrigues, P Matafome, C Amaral, E Munes, T Louro, P Monteiro and R Seiça, unpublished results) and could result from the adaptation in their selection process. This inflammatory marker, produced in liver and linked with insulin-resistance ${ }^{(21)}$, did not change with dietary restriction in both strains. In relation to muscular inflammatory biomarkers, IL-6 levels were higher in GK rats. The levels of TNF- $\alpha$ and the expression of normal and phosphorylated form of SAPK/JNK were similar in both strains.

Dietary restriction in diabetic rats was able to reduce the skeletal muscle inflammatory process and to improve glucose tolerance, insulin resistance, NEFA and HbA1c levels and adipokine profile but aggravated fasting glycaemia. This effect on glycaemia was also observed in the WDR group, suggesting a higher rate of hepatic glucose production in the fasting state, as a result of severe dietary restriction. Furthermore, dietary restriction in normal rats reduced body weight, NEFA and leptin levels, and raised adiponectinaemia. In other parameters, the levels did not change, and the levels of IL-6 even increased. Based on this, future studies are required in 

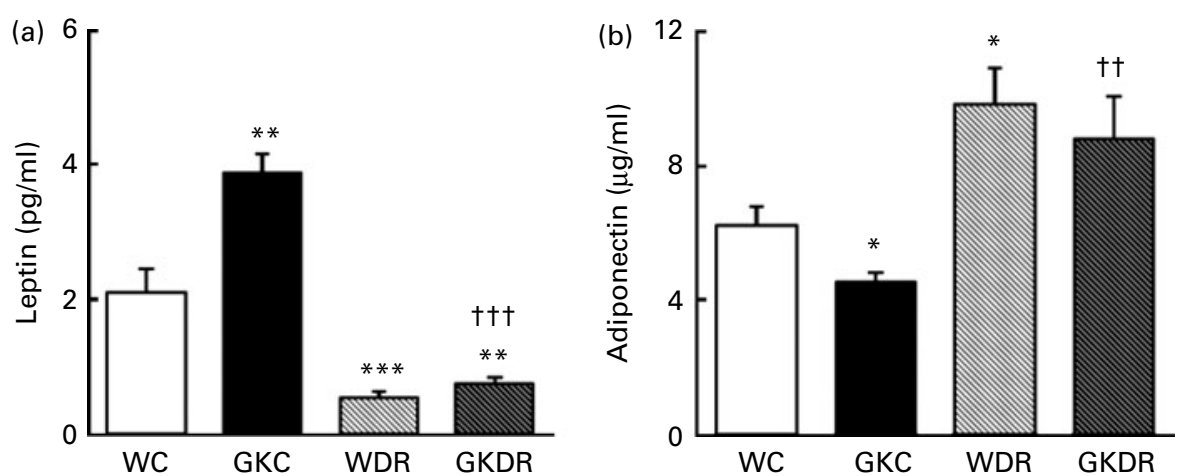

Fig. 3. Effects of dietary restriction on (a) plasma levels of leptin and (b) serum levels of adiponectin in Wistar and Goto-Kakizaki (GK) rats. WC, Wistar control; GKC, GK control; WDR, Wistar rats with dietary restriction; GKDR, GK rats with dietary restriction. Values are means for nine to thirteen rats per group, with standard errors represented by vertical bars. Mean value was significantly different from that of the WC group: ${ }^{*} P<0.05,{ }^{\star *} P<0.01$, ${ }^{\star * *} P<0.001$. Mean value was significantly different from that of the GKC group: †† $P<0.01, \dagger^{+}+P<0.001$

order to evaluate the impact of such pronounced dietary restriction on inflammation in normal models. The adipose tissue modulates its own metabolism but is also able to interfere in other tissues, in order to regulate its metabolism. Its secretory products are involved in glucose and lipid homeostasis and inflammation. In fact, it has become recently clear that adipocytes represent active secretory cells. The adipocytes were able to release NEFA by lipolysis and produce a variety of cytokines ${ }^{(2,11,12)}$. Adipocyte dysfunction leads to excessive fat accumulation in non-adipose tissue and disturbed adipokine secretion ${ }^{(20)}$, which may have local and systemic effects ${ }^{(10)}$. Adipose tissue in insulin resistance is characterised by increased levels of leptin and proinflammatory cytokines such as TNF- $\alpha$ and IL-6 and lower adiponectin expression $^{(2,4,10)}$.

Leptin, whose levels are proportional to overall adipose mass $^{(4)}$, has important effects on peripheral tissue including muscle $^{(22)}$, but its effects are still controversial ${ }^{(23)}$. In some studies leptin has increased insulin sensitivity but others have shown, however, that this adipokine impairs insulin action $^{(2)}$. Furthermore, in obese and diabetic states, it is likely that higher levels of leptin are indicative of leptin resistance $^{(11,23)}$. In the present study we verified that GK rats have higher levels of leptin, showing the previously reported leptin resistance in this animal model ${ }^{(19)}$ that decreased after dietary restriction.

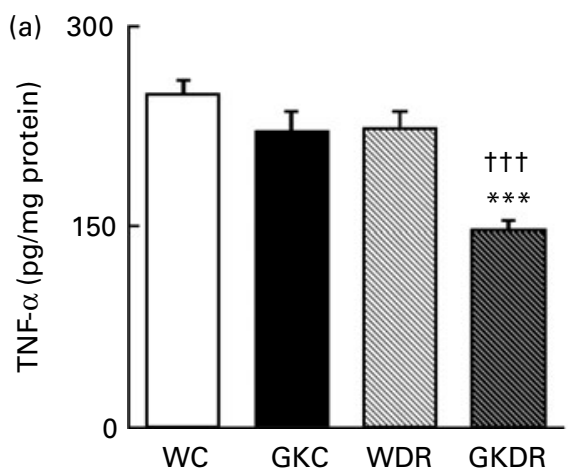

A recent report suggests that adiponectin has antidiabetic properties such as stimulating glucose uptake in skeletal muscle and suppressing hepatic glucose production. Antiinflammatory properties have also been reported ${ }^{(24)}$. Adiponectin levels correlate inversely with the impairment of insulin signalling; it decreases hyperglycaemia and NEFA and affects immune and inflammatory processes throughout the body ${ }^{(23)}$. Our data show that the levels of adiponectin, a positive adipokine, increased with dietary restriction in normal and diabetic rats. AdipoR1 expression predominates in skeletal muscle ${ }^{(14)}$. It has also been described that adiponectin antagonises many effects of TNF- $\alpha$ and its production is decreased by this inflammatory factor ${ }^{(25)}$. Therefore, the increase of adiponectin levels induced by dietary restriction may justify the improvement of TNF- $\alpha$ and IL-6 levels in skeletal muscle of diabetic rats and may be associated with the amelioration of insulin resistance and metabolic profile.

Plasma TNF- $\alpha$ and IL-6 are commonly elevated in type 2 diabetes $^{(2,8)}$ and have a negative effect on insulin signalling, although the effect of IL-6 in skeletal muscle remains unclear $^{(2,8,14,23)}$.

$\mathrm{TNF}-\alpha$ impairs insulin sensitivity in rodent skeletal muscle ${ }^{(10)}$. While the mechanism underlying desensitisation has not been fully elucidated, TNF- $\alpha$ decreases tyrosine phosphorylation of insulin receptor substrate (IRS)-1 and increases IRS-1 serine phosphorylation, and an improvement

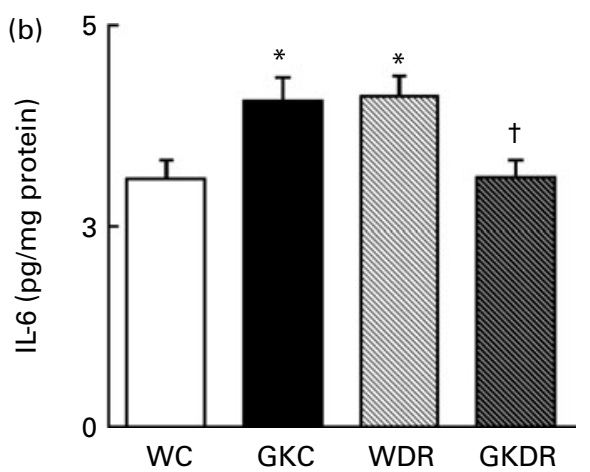

Fig. 4. Effects of dietary restriction on muscle levels of (a) TNF- $\alpha$ and (b) IL-6 in Wistar and Goto-Kakizaki (GK) rats. WC, Wistar control; GKC, GK control; WDR, Wistar rats with dietary restriction; GKDR, GK rats with dietary restriction. Values are means for nine to thirteen rats per group, with standard errors represented by vertical bars. Mean value was significantly different from that of the WC group: ${ }^{*} P<0.05$, ${ }^{\star * *} P<0.001$. Mean value was significantly different from that of the GKC group: $\uparrow P<0.05$, $† \dagger \dagger P<0.001$. 
(a)
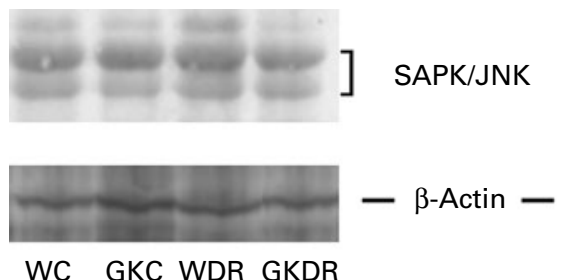

(c)
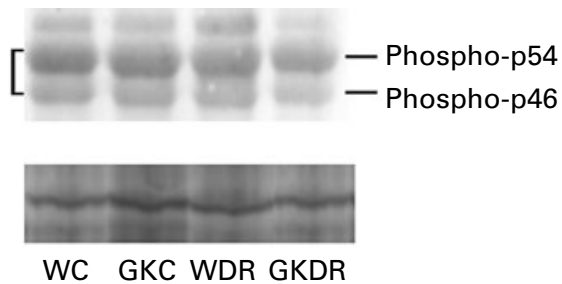

(b)

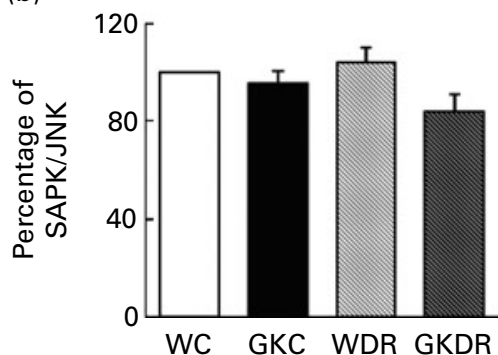

(d)

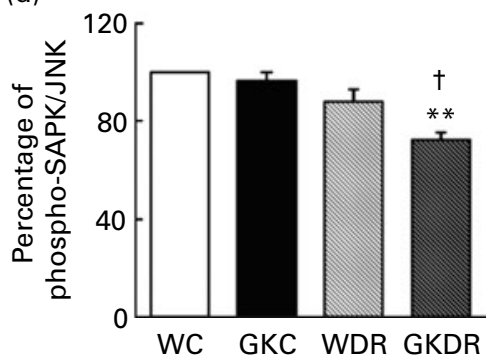

Fig. 5. Effects of dietary restriction on (a, b) stress-activated protein kinase (SAPK)/Jun-N-terminal kinase (JNK) and (c, d) phospho-SAPK/JNK expression in muscular tissue in Wistar and Goto-Kakizaki (GK) rats. WC, Wistar control; GKC, GK control; WDR, Wistar rats with dietary restriction; GKDR, GK rats with dietary restriction. $(\mathrm{a}, \mathrm{c})$ Representative Western blots. (b, d) Percentage of expression of proteins in relation to the WC group by quantification of the bands using the ImageQuant program (Molecular Dynamics, Sunnyvale, CA, USA). Values are means for nine to thirteen rats per group, with standard errors represented by vertical bars. ${ }^{* *}$ Mean value was significantly different from that of the WC group $(P<0.01)$. † Mean value was significantly different from that of the GKC group $(P<0.05)$.

of insulin receptor phosphorylation in skeletal muscle is achieved with an anti-TNF- $\alpha$ antibody ${ }^{(8)}$. Moreover, TNF- $\alpha$ also activates an apoptotic signalling pathway that can degrade the muscle tissue and energy restriction attenuates the loss of muscle mass and function intervening on TNF- $\alpha$ signalling ${ }^{(8,26)}$.

IL-6 can have distinct effects on different tissues. There are discrepancies between acute and long-term effects and its action depends on complex interactions with other pro- and anti-inflammatory cytokines ${ }^{(8)}$. In a recent study, physical exercise is associated with increased insulin action in skeletal muscle and with increased IL- 6 circulating levels. The authors suggest a possible anti-inflammatory action of IL-6 in skeletal muscle ${ }^{(14)}$, but the real effect remains controversial. In most studies about diabetes, IL-6 seems to impair insulin action $^{(2,8,14,23)}$. Accordingly, with these studies, the present results show that muscle IL-6 levels were higher in diabetic rats. Dietary restriction was able to reduce both pro-inflammatory cytokines (IL- 6 and TNF- $\alpha$ ), and these observations may be related to the improvement in insulin resistance indexes after the diet.

Various studies reported that increased circulating plasma levels of TAG and NEFA contribute to insulin resistance in peripheral tissues such as skeletal muscle $e^{(2,7,27)}$. Furthermore, it was observed that dietary restriction increases mitochondrial biogenesis and NEFA oxidation ${ }^{(28)}$. The reduction of NEFA in GK rats induced by dietary restriction may be also related with improvement of insulin resistance.

High levels of fatty acids and pro-inflammatory cytokines activate JNK in liver, adipocyte cells and skeletal muscle, resulting in insulin-sensitivity reduction ${ }^{(20)}$.

JNK is a mitogen-activated protein kinase and some previous studies made clear the role of JNK in inflammation and metabolic diseases, suggesting JNK inhibition as a viable therapeutic intervention in diabetes mellitus and atherosclerosis ${ }^{(3)}$.
JNK is activated by pro-inflammatory cytokines such as TNF- $\alpha$ and plays a negative role in insulin signalling ${ }^{(2,3,5)}$. The activated form, phosphorylated JNK, mediates IRS-1 serine phosphorylation and this phosphorylation can induce the dissociation of IRS proteins from the insulin receptor, impairing insulin signalling ${ }^{(2,3,5)}$. Our data show that dietary restriction in diabetic animals decreased the expression of the active form of $\mathrm{JNK}$, and this can be related to the reduction of TNF- $\alpha$ and NEFA levels and the improvement of the hormone profile.

Energy restriction might affect glucose tolerance, modulating insulin action in skeletal muscle, and inducing elevated insulin-stimulated glucose transport in this tissue ${ }^{(29-31)}$. The improvement of glucose tolerance and HbAlc in GK rats is in accordance with these studies.

In conclusion, dietary restriction in type 2 diabetes has a central role in adipocyte stress, influencing adipokines and other proinflammatory mediator factors and energy balance, giving rise to improved skeletal muscle inflammation and insulin sensitivity.

\section{Acknowledgements}

We thank Mário Simões from the Institute of Physiology, Faculty of Medicine of Coimbra for his technical support. We also thank Serviço de Patologia Clínica from the University Hospital of Coimbra for technical support.

The present study was supported by the University of Coimbra and University Hospitals of Coimbra. We thank SERVIER Portugal for financial support.

J. C. was the main contributor to the practical work pertaining to the present paper. L. R., P. Matafome, C. A., E. N. and T. L. contributed with technical support, including animal management and treatment. P. Monteiro and R. S. contributed with scientific advice.

There are no potential conflicts of interest. 


\section{References}

1. Wilde S, Roglic G, Green A, et al. (2004) Global prevalence of diadetes: estimates for the year 2000 and projections for 2030 . Diabetes Care 27, 1047-1053.

2. Sell H, Eckel J \& Dietze-Schroeder D (2006) Pathways leading to muscle insulin resistance - the muscle-fat connection. Arch Physiol Biochem 112, 105-113.

3. Wellen KE \& Hotamisligil GS (2005) Inflammation, stress, and diabetes. J Clin Invest 115, 1111-1119.

4. Sesti G (2006) Pathophysiology of insulin resistance. Best Pract Res Clin Endocrinol Metab 20, 665-679.

5. Bastard JP, Maachi M, Lagathu C, et al. (2006) Recent advances in the relationship between obesity, inflammation, and insulin resistance. Eur Cytokine Netw 17, 4-12.

6. Ugochukwu N \& Figgers C (2007) Caloric restriction inhibits up-regulation of inflammatory cytokines and TNF- $\alpha$, and activates IL-10 and haptoglobin in the plasma of streptozotocininduced diabetic rats. $J$ Nutr Biochem 18, 120-126.

7. Schenk S, Saberi M \& Olefsky JM (2008) Insulin sensitivity: modulation by nutrients and inflammation. $J$ Clin Invest 118, 2992-3002.

8. Wei Y, Chen K, Whaley-Connell AT, et al. (2008) Skeletal muscle insulin resistance: role of inflammatory cytokines and reactive oxygen species. Am J Physiol Regul Integr Comp Physiol 294, R673-R680.

9. Musi N \& Goodyear LJ (2006) Insulin resistance and improvements in signal transduction. Endocrine 29, 73-80.

10. Goossens GH (2007) The role of adipose tissue dysfunction in the pathogenesis of obesity-related insulin resistance. Phyisiol Behav 94, 206-218.

11. Rabe K, Lehrke M, Parhofer KG, et al. (2008) Adipokines and insulin resistance. Mol Med 14, 741-751.

12. Guerre-Millo M (2004) Adipose tissue and adipokines: for better or worse. Diabetes Metab 30, 13-19.

13. Rasouli N \& Kern PA (2008) Apopocytokines and the metabolic complications of obesity. J Clin Endocrinol Metab 93, S64-S73.

14. Roth GS, Ingram DK \& Lane MA (2001) Caloric restriction in primates and relevance to humans. Ann N Y Acad Sci 928 , $305-315$.

15. Fontana L \& Klein S (2007) Aging, adiposity, and caloric restriction. JAMA 297, 986-994.

16. American Diabetes Association (2008) Nutrition recommendations and interventions for diabetes: a position statement of the American Diabetes Association. Diabetes Care 31, S61-S78.
17. Nunes E, Peixoto F, Louro T, et al. (2007) Soybean oil treatment impairs glucose-stimulated insulin secretion and changes fatty acids composition of normal and diabetic islets. Acta Diabetol 44, 121-130.

18. Seiça R, Santos DL, Palmeira CM, et al. (2005) Mitochondrial function is not affected by renal morphological changes in diabetic Goto-Kakizaki rat. Toxicol Mech Methods 15, 253-261.

19. Maekawa F, Fujiwara K, Kohno D, et al. (2006) Young adultspecific hyperphagia in diabetic Goto-Kakizaki rats is associated with leptin resistance and elevation of neuropeptide Y mRNA in the arcuate nucleus. J Neuroendocrinol 18, 748-756.

20. Ferranti S \& Mozaffarian D (2008) The perfect storm: obesity, adipocyte dysfunction, and metabolic consequences. Clin Chem 54, 945-955.

21. Ndumele CE, Pradhan AD \& Ridker PM (2006) Interrelationships between inflammation, C-reactive protein, and insulin resistance. J Cardiometab Syndr 1, 190-196.

22. Toyoshima Y, Gavriola O, Yakar S, et al. (2005) Leptin improves insulin resistance and hyperglycemia in a mouse model of type 2 diabetes. Endocrinology 146, 4024-4035.

23. Muoio DM \& Newgard CB (2008) Molecular and metabolic mechanisms of insulin resistance and $\beta$-cell failure in type 2 diabetes. Nat Rev Mol Cell Biol 9, 193-205.

24. Sweeney FG (2006) Mechanisms regulating energy metabolism by adipoectin in obesity and diabetes. Biochem Soc Trans 34, 5 .

25. Whitehead JP, Richards AA, Hickman IJ, et al. (2006) Adiponectin - a key adipokine in the metabolic syndrome. Diabetes Obes Metab 8, 264-280.

26. Dirks AJ \& Leeuwenburgh C (2006) Tumor necrosis factor $\alpha$ signalling in skeletal muscle: effects of age and caloric restriction. J Nutr Biochem 17, 501-508.

27. Petersen KF \& Shulmam GI (2006) Etiology of insulin resistance. Am J Med 119, 10S-16S.

28. Lange P, Moreno M, Silvestri E, et al. (2007) Fuel economy in food-deprived skeletal muscle: signaling pathways and regulatory mechanisms. FASEB $J$ 21, 3431-3441.

29. Park S, Komatsu T, Hayashi H, et al. (2006) Caloric restriction at middle age improved glucose tolerance without affecting age-related impairments of insulin signalling in rat skeletal muscle. Exp Gerontol 41, 837-845.

30. Arias EB \& Cartee GD (2007) In vitro simulation of caloric restriction-induced decline in glucose and insulin leads to increase insulin-stimulated glucose transport in rat skeletal muscle. Am J Physiol Endocrinol Metab 293, E1782-E1788.

31. Dean DJ, Brozinick JT, Cushman SW, et al. (1998) Calorie restriction increases cell surface GLUT-4 in insulin-stimulated skeletal muscle. Am J Physiol 275, E957-E964. 